\title{
Statistical Mechanisms for Detecting Malicious Behaviors in Resource Allocation from Non- cooperative P2P Environments
}

\author{
Hai-yang Hu* \\ School of Computer Science, Hangzhou Dianzi University, \\ Hangzhou, 310018, China \\ State Key Laboratory for Novel Software Technology, Nanjing university, \\ Nanjing, 210093, China \\ Zhong-jin Li \\ School of Computer Science, Hangzhou Dianzi University, \\ Hangzhou, 310018, China \\ Li-guo Huang \\ Department of Computer Science, Southern Methodist University, \\ Dallas, 75275-0122, USA \\ Hua Hu \\ School of Computer Science, Hangzhou Dianzi University, \\ Hangzhou, 310018, China \\ Received 7 November 2011 \\ Accepted 15 June 2012
}

\begin{abstract}
A Peer-to-Peer (P2P) system relies on the cooperation of the peers and the contributions of their resources. To motivate autonomous peers share their resources, the system needs to support effective resource allocation strategies with respect to peers' task priorities, and their personal information about valuation. However, peers may tend to be selfish for saving their limited resources and act as the free-riders. Some peers may even be malicious with the goal to do damage to the system. In this paper, we present a bidding based approach for resource allocation to address these issues. We investigate peers' bidding strategies under different scenarios in terms of probability distributions that peers' valuations of their prioritized tasks follow in achieving the Nash equilibrium. For resisting the damage to the P2P system brought by malicious peers, we explore different types of malicious behavior and present several statistical mechanisms to detect the malicious peers. The algorithm is also presented for bidders performing their auctions. Finally, we conducted experiments to show the effectiveness of the proposed mechanisms.
\end{abstract}

Keywords: Peer-to-Peer system; detecting malicious behavior; statistical mechanism; resource allocation.

\section{Introduction}

PEER-TO-PEER networks (P2P) have emerged as new technologies in distributed applications, mainly due to the advances in network, file sharing system, and distributed systems technologies ${ }^{1}$. P2P systems have increased dramatically in its size, use, and underlying

\footnotetext{
* Corresponding author: huhaiyang@hdu.edu.cn
} 
protocols, and it has been continuously focused on by researchers and engineers.

To implement an effective and secure resource allocation in $\mathrm{P} 2 \mathrm{P}$ computing systems, several challenges must be addressed. First, as P2P systems are open and dynamic, they incorporate different types of peers with their private valuations for the public resources and multi-priority tasks, which should be considered when designing effective resource allocation schemes for better sharing of resources. In addition, peers could be "selfish" for maximizing their own utility. Moreover, some peers may even be malicious and aim at doing damage to the system and other peers. Therefore, some special mechanisms are needed to detect these malicious peers and resist their harmful behavior.

Recently, there have been many mechanisms proposed for resource allocation in different types of computing paradigms ${ }^{5-15}$. They can be categorized into three groups ${ }^{1}$ : market-based, reciprocity-based and reputation-based approaches. In the market-based approaches ${ }^{5-7,11}$, peers submit bids for resources. Then, the resources are allocated due to some predefined resource allocation mechanisms.

In the reciprocity-based approaches ${ }^{12-14}$, each peer records and evaluates the behaviors of others that it interacts with, and then makes decision on how to serve another one by the direct service exchanges it receives from this peer. Moreover, in the methods by using reputation-based approaches ${ }^{8-10}$, the reputation of each peer is derived from its behavioral histories of providing services and consuming services. A peer having a good behavioral history of serving other ones is provided a service with better quality, when it competes with other users for some resources. Meanwhile, game theoretic methods $^{14,15}$ have also been used to mathematically model cooperation in P2P networks. In these methods, the authors attempted to make peers achieve a Nash equilibrium (NE) state under which no one can improve their utility any more by deviating from its current strategy. However, we note that the reciprocity-based and reputation-based approaches may not be very applicable to time-critical P2P computing systems, and it needs the system running for an enough long time.

In this paper, we propose to achieve the goal of effective and secure resource allocation in $\mathrm{P} 2 \mathrm{P}$ computing environments by designing a bidding-based method under the game theoretic framework. We start by studying a general scenario under which multiple peers bid for a group of resources simultaneously, and derive the Nash equilibrium solutions. In designing an anti-cheating resource allocation mechanism for peers, we explore different types of cheating behaviors, and then propose several statistics-based mechanisms for detecting the malicious peers from the non-cooperative environments. Finally, we conduct the experiments to show the effectiveness of the proposed methods.

This paper makes the following major contributions.

- We address the issue of resource allocation in P2P computing environments by incorporating different types of peers with varied private valuations for the resources and multi-priority tasks, and then derive peers' bidding strategies in achieving NE state.

- We explore different types of cheating behaviors that could be demonstrated by malicious peers during the process of bidding for resources, and then discuss several statistics-based mechanisms to distinguish them from the non-cooperative environment.

- We have made an experimental study on our detecting methods. We evaluate the peers' payoff under different scenarios, and find that our detecting methods can effectively improve their payoff and reduce the malicious bidding behaviors.

The rest of the paper is organized as follows: Section 2 gives an overview on the related works. Section 3 introduces our resource allocation game model among peers and the major notations used in this paper. Section 4 explores the peers' cheating strategies and presents several statistics-based mechanisms for detecting their malicious behaviors. The algorithm is given in Section 5 to help peers make their bidding decisions. Section 6 conducts extensive experiments to evaluate the effectiveness of the proposed methods. Finally, Section 7 briefly concludes the work presented in this paper.

\section{Related Works}

Recently, there are numerous mechanisms proposed for resource allocation in peer-to-peer environments. To preserve effective resource allocation among peers, existing methods can be roughly classified into three main approaches, market-based approaches, reciprocitybased approaches, and reputation-based approaches. These methods usually use the game theoretic technique to derive their solutions. 


\subsection{Market-based approaches}

In the market-based approach, peers submit bids for resources, and the resources are allocated according to some predefined resource allocation mechanisms.

In Ref.7, the authors proposed a payment-based incentive mechanism to cope with the problem of media streaming in the $\mathrm{P} 2 \mathrm{P}$ network. Peers can earn points through contributing their resources to the P2P system, and then use these points to bid for a good source node in the next time period. Thus, peers will receive different media qualities by making different contributions to the P2P network.

In Ref.11, the authors proposed a proportional-share mechanism to allocate resources among the users. Under this mechanism, each peer partitioned its budget among the multiple resources and receives a fraction of each resource proportional to its bid.

In Ref.16, the authors used a single type of currency and a set of banks to facilitate secure trading. A peer's wealth was increased when its resources were contributed and decreased when they were consumed.

\subsection{Reciprocity-based approaches}

In reciprocity-based approaches, a peer monitored other peers' behaviors and evaluated their contribution levels, which can be defined as a numerical assessment of peers' contribution to the P2P network. These approaches usually involve a mutual action, i.e., the service quality that a peer received was determined by its contribution level.

In Ref.14, the authors proposed a reciprocity-based incentive mechanism named Global Contribution to solve the problem of resource sharing in P2P networks. The authors introduced a popular tit-for-tat policy to keep the fairness of bandwidth contribution to the networks.

In Ref.17, the authors considered altruism as a key element of P2P streaming broadcast. They showed that the level of altruism has an important impact on the overlay. Even a small degree of altruism can bring significant benefits to the overall system performance.

In Ref.18, the authors proposed a taxation model, in which resource-rich peers were required to contribute more bandwidth to the system and subsidize the resource-poor peers. The social welfare was hence improved through the redistribution of wealth (that is, individual benefits in terms of the received media rate).

\subsection{Reputation-based approaches}

Reputation-based approaches construct and maintain reputation information about peers, and peers with good reputations are offered with better services. The reputation information about a peer is on the basis of feedback from other peers who have interacted with this peer. Such feedback can be positive or negative. The system uses the feedback to build up a good reputation for contributing peers and a bad one for free riders.

A reputation-based method for resource allocation in P2P system was presented in Ref.10. The authors thought that every peer had a capacity-limited bandwidth for its upstream and downstream connections. They used the reputation-based method to increase peers' reputation by contributing their resources to others, and peers made use of their reputation for downloading.

In Ref.8, the authors proposed a ranking-based method for resource allocation. They presented a theoretic framework for optimal resource allocation and admission control for peer-to-peer networks. Peer's behavioral ranks were incorporated into the resource allocation, and the admission control was used to provide differentiated services and can block peers with bad ranks.

Some works on reputation-based systems ${ }^{9}$ provided incentive mechanisms to enforce collaboration between peers by controlling not only the provider selection policy but also the client selection policy.

However, we note that in time-critical P2P computing systems, some peers may need to obtain resources in a real-time manner. As the reciprocitybased and reputation-based approaches need the system running for long enough time, they may not be very applicable to these applications. On the other hand, current works based on market-based mechanisms mainly focus on resource allocation among peers in a relatively static environment, while we concentrate on an open, dynamic and non-cooperative P2P environment where peers can dynamically join or leave the system, their tasks can be generated with different priorities, and the number of resources and tasks are continuously changing with time elapsing. Meanwhile, some peers may even perform malicious behaviors. Thus, we also propose several statistical mechanisms for detecting out the malicious peers from this noncooperative environment. In other words, existing works may complement well with our work in this aspect. 


\section{Resource Allocation}

In this paper, each resource, such as peer's CPU cycles and the action to transmit a data packet for others, is treated as a discrete good and can be provided to environments by peers through auctions. When a peer needs resources to finish its tasks, it submits bids for these resources and competes with other buyers. Each peer autonomously generates its own tasks with different levels of priorities. Thus, the peers' valuation of the same resource may be quite different according to their prioritized tasks. For example, if a peer holds a high-priority task, it will be inclined to pay more bids for the resource to finish the task. On the contrary, if the peer needs to finish a low-priority task, it may submit lower bids than its competitors do. In the following of this section, we will discuss the resource allocation game (RAG) for peers' prioritized tasks in details. We use the first-price sealed-bids auction ${ }^{2}$ in the game, i.e., when several peers compete for one resource, the peer who submits the highest bid becomes the winner and obtains this resource.

The major notations used in this paper are given in Table 1

Table 1. Definitions of major notations

\begin{tabular}{|c|c|}
\hline Notation & Definition \\
\hline$A_{i}(t)=\left\{a_{i}^{m}\right\}$ & The set of tasks generated by $p a_{i}$ in stage $t$ \\
\hline$P A=\left\{p a_{i}\right\}$ & The set of peers in current environments \\
\hline$v_{i}^{m}$ & $p a_{i}{ }^{\prime} s$ private valuation for its task $a_{i}^{m}$ \\
\hline$b_{i}^{m}$ & $\begin{array}{l}\text { The corresponding bid submitted by } p a_{i} \text { to obtain a } \\
\text { resource so as to finish its task } a_{i}^{m}\end{array}$ \\
\hline$R_{i}(t)=\left\{r_{i}^{k}\right\}$ & Resources that can be contributed by $p a_{i}$ in stage $t$ \\
\hline$c_{i}(t)$ & The number of tasks generated by $p a_{i}$ in stage $t$ \\
\hline$d_{i}(t)$ & $\begin{array}{l}\text { The number of available resources contributed } \\
\text { by } p a_{i} \text { in stage } t\end{array}$ \\
\hline$g_{i}^{k}$ & $\begin{array}{l}\text { The payoff gained by } p a_{i} \text { through selling its } k \text {-th } \\
\text { resource by auction in stage } t\end{array}$ \\
\hline$e\left(a_{i}^{m}, r_{j}^{n}\right)$ & $\begin{array}{l}\text { The payoff gained by } p a_{i} \text { after it obtains } \\
\text { resource } r_{j}^{n} \text { and finish the task } a_{i}^{m}\end{array}$ \\
\hline
\end{tabular}

\subsection{Game model}

We use the bidding mechanism proposed in Ref.2 to model our resources allocation game. In our resources allocation game, each task $a_{i}$ generated by a peer $p a_{i}$ has a priority denoted by $\operatorname{priority}\left(a_{i}\right)$. We also use $v_{i}$ to denote $p a_{i}$ 's monetary valuation for $a_{i}$ in terms of its $\operatorname{priority}\left(a_{i}\right)$, which is typically application-specific and remains unknown by its competitors. Suppose now a resource $r$ is provided to a group of peers, and it can be used to finish their tasks. Each $p a_{i}$ will have a different valuation of $r$ according to the evaluation of its own task $a_{i}$. Without losing generality, we use $v_{i}$ to denote $p a_{i}$ 's monetary valuation of $r$ in finishing its task $a_{i}$. In this paper, we focus on achieving a symmetric Nash equilibrium, where the bidding function $\beta$ is the same to all the peers. The bid submitted by $p a_{i}$ for $r$ is defined by $b_{i}=\beta\left(v_{i}\right)$.

First, we introduce our resource allocation game (RAG). In such a game, peers' valuations are at least $\underline{v}$ with $\underline{v} \geq 0$, and at most $\bar{v}$, which is a common knowledge known by all peers. Thus, $[v, \bar{v}]$ is the interval over which each peer's valuation $v_{i}$ ranges. Let $N$ represent the number of peers in a resource allocation game, and $S_{i}=\left\{s_{i} \mid s_{i} \in[\underline{v}, \bar{v}]\right\}$ be the bidding strategy space of peer $p a_{i}$, then the bidding strategy combination of all peers can be denoted by $s=\left(s_{1}, \cdots, s_{N}\right)$, where $S=\times_{1 \leq i \leq N} S_{i}$ is the peers' strategy space. Furthermore we use $s_{-i}$ to denote the strategy combination of all peers except $p a_{i}$, i.e.,

$$
s_{-i}=\left(s_{1}, \cdots, s_{i-1}, s_{i+1}, \cdots s_{N}\right)
$$

Next, we use $U_{i}(s)$ to denote the utility of $p a_{i}$ under the strategy combination $s$. Finally, a strategy combination, $s^{*}$, is said to achieve the state of Nash equilibrium, if and only if the following condition holds:

$$
U_{i}\left(s^{*}\right) \geq U_{i}\left(s_{-i}^{*}, s_{i}\right) \forall s_{i} \in S_{i}, 1 \leq i \leq N
$$

Note that, if a strategy combination is said to achieve the state of Nash equilibrium, then no peer can improve its utility by unilaterally deviating from its own strategy.

\section{2. $N$-peer resource allocation game}

We study a general scenario where there are $N(N>2)$ peers bidding for a public resource $r$, which we call a $N$-peer resource allocation game. Assume that in stage $t$, each $p a_{i}$ holds a set of tasks $A_{i}(t)=\left\{a_{i}^{m}\right\}$ with each $a_{i}^{m}$ having an valuation $v_{i}^{m}$. Let $c_{i}(t)=\left|A_{i}(t)\right|$ be the number of these tasks. Then, for each task $a_{i}^{m} \in A_{i}(t), p a_{i}$ needs to submit a bid $b_{i}^{m}$ to get a necessary resource so as to finish it. Thus, the total amount of payoff gained by $p a_{i}$ with bidding for resources to finish its tasks in stage $t$, can be given by

$$
E_{i}(t)=\sum_{m=1}^{c_{i}(t)}\left(v_{i}^{m}-b_{i}^{m}\right) p_{i}^{m}
$$


where $p_{i}^{m}$ is used to denote whether $p a_{i}$ can successfully obtain a resource to finish its task $a_{i}^{m}$, i.e., if $p a_{i}$ can do this, $p_{i}^{m}=1$; otherwise, $p_{i}^{m}=0$.

Let $F_{i}(x)$ be the probability distribution function that $p a_{i}{ }^{\prime} s$ valuation for resources follows on $[v, \bar{v}]$, which can be obtained from its data logs over a long period of time. Without losing generality, it is assumed that $F_{i}(x)$ is continuous on $[v, \bar{v}]$, and is defined by

$$
F_{i}(V)=\operatorname{Pr}\left(v_{i} \leq V\right)=\int_{V}^{V} f_{i}(x) d x
$$

where $V \leq \bar{v}$ and $f_{i}(x)$ is the corresponding probability density function (pdf) of $F_{i}(x)$ on $[v, \bar{v}]$.

In the auction for $r$, each peer is required to report its $F_{i}\left(v_{i}\right)$ to the resource provider. Then, resource provider broadcasts the information of $\left\{F_{i}(x)\right\}$ to all the peers currently participating in the auction so as to help them develop the bidding strategies. In addition, we note that some peer may misreport its $F_{i}(x)$. A statistics-based mechanism is given in Section 4.1 for checking whether the peers honestly report their $F_{i}(x)$.

On the other hand, each $p a_{i}$ can also play a role of resource provider, i.e., in stage $t, p a_{i}$ may have a group of available resources $R_{i}(t)=\left\{r_{i}^{k}\right\}$, and it can sell them out by auction to other peers. Let $g_{i}^{k}$ denote the payoff gained by $p a_{i}$ through selling out a resource $r_{i}^{k}$ in stage $t$, and $d_{i}(t)=\left|R_{i}(t)\right|$ be the number of resources. Then, $G_{i}(t)$, the total amount of payoff gained by $p a_{i}$ through selling its resources in stage $t$, is given by

$$
G_{i}(t)=\sum_{k=1}^{d_{i}(t)} g_{i}^{k} q_{i}^{k}
$$

where $q_{i}^{k}$ is used to denote whether $p a_{i}$ successfully sells out its resource $r_{i}^{k}$, i.e., if $q_{i}^{k}=1$, then $r_{i}^{k}$ is sold out; otherwise, $q_{i}^{k}=0$.

In this way, the total amount of utility gained by $p a_{i}$ in stage $t$ can be given by:

$$
U_{i}(t)=E_{i}(t)+G_{i}(t)
$$

We give the bidding strategy for peers in achieving the state of a symmetric Nash equilibrium in a $N$-peer RAG, and the optimal bidding strategy is given as follows.

Theorem 1. In the $N$-peer $R A G$, let $F_{i}(x)$ represent the probability distribution that $p a_{i}$ 's valuation follows. Then, the optimal bidding strategy for the peers in achieving the state of a symmetric Nash equilibrium is $b^{*}=\left(b_{1}^{*}, b_{2}^{*}, \cdots, b_{N}^{*}\right)$ where

$$
b_{i}^{*}=\beta\left(v_{i}\right)=v_{i}-\frac{\int_{\underline{v}}^{v_{i}} \prod_{j=1, j \neq i}^{n} F_{j}(x) d x}{\prod_{j=1, j \neq i}^{n} F_{j}\left(v_{i}\right)}
$$

Note that theorem 1 is different from the conclusion presented in Ref.2. In theorem 1, the $\left\{F_{i}(x)\right\}$ of peers can be different, while in Ref.2, they are required to be identical. RAG

Next, we analyze the existence of NE in the $\mathrm{N}$-peer

Theorem 2. In the $N$-peer RAG, there exists the Nash equilibrium.

Finally, we also present another property in this $\mathrm{N}$ peer $\mathrm{RAG}^{2}$ :

Theorem 3. In $N$-peer RAG with private valuations, each $p a_{i}{ }^{\prime} s$ optimal bid $b_{i}^{*}$ rises in price with the increasing number of its competitors.

\section{Malicious-Behavior Detection Mechanisms}

Because P2P computing environments are dynamic and open, some peers may be malicious with the goal to do damage to the system. In this section, we focus on studying malicious behaviors inside the system (i.e., these peers also have legitimate identities), and our goal is to design several detection mechanisms to distinguish these malicious peers from other benign ones in the environments.

In this section, we focus on the scenario where the system containing of a finite number of peers will keep running for an enough long time. Each peer will stay in the system for a reasonably long time. We also assume that each peer has a unique registered identity, which can be verified by a central authority (CA). This identification will be used to perform necessary access control and authentication. CA also manages the information of all the auctions occurring in the system, i.e., after a successful auction, the resource provider reports the details of the auction process to $\mathrm{CA}$, which includes the identities of the bidders participating in the auction, their bids and the final winner. Thus, CA can use this information to detect the malicious peers. In addition, there exists a central bank (CB) in the system, which stores each peer's virtual currency, deposit and transaction. It also fairly exchanges virtual currency between resource providers and bidders.

To provide an effective resource allocation mechanism among selfish peers in a hostile environment, several statistical mechanisms are presented for detecting peers' malicious behaviors. 
For a malicious $p a_{i}$, its goal is to increase its own utility by degrading the system performance and wasting the resources of other peers as much as possible. In general, $p a_{i}$ can achieve its goal by taking the following harmful actions:

- $p a_{i}$ misreports its $F_{i}(x)$. In the RAG, a rational $p a_{i}$ can derive its optimal bidding strategy $b_{i}^{*}$ according to theorem 1 . It needs to know in advance the $\left\{F_{i}(x)\right\}$ of other peers who also join in the auction. Thus, in our RAG, each $p a_{i}$ must honestly report its $F_{i}(x)$ to the resource provider. However, some malicious peers may misreport their $F_{i}(x)$ to disturb other peers' bidding strategies and do damage to their utilities. We need some approaches to detect this kind of cheating behavior.

- $p a_{i}$ excessively bids for resources to accomplish its own tasks. A malicious peer may also extremely bid for as many resources as possible, in order to increase the probability of obtaining the resources, and at the same time to increase the bids paid by other peers (seen from Theorem 3). For example, in stage $t$, a malicious $p a_{i}$ generates five tasks, $a_{i}^{1}, \ldots, a_{i}^{5}$. It submits bids for ten available resources in the environments, and obtains eight resources. By carefully choosing five resources which have lower prices and dropping the other three resources, $p a_{i}$ enhances its payoff.

\subsection{Peer misreports its $F_{i}(x)$}

In an auction, each rational peer can derive its optimal bidding function from the $\left\{F_{i}(x)\right\}$ of other peers according to theorem 1 . The system needs each peer to honestly report its $F_{i}(x)$ to the resource provider and CA. However, since some malicious peers may misreport their $F_{i}(x)$, we need to devise a method to detect their cheating behaviors. Based on chi-square goodness-of-fit test ${ }^{3}$, a mechanism called $D M_{F}$ is discussed in this section to detect this type of cheating behavior. The idea of $D M_{F}$ is to check whether $p a_{i}{ }^{\prime} s$ actual bidding behavior is consistent with its $F_{i}(x)$ reported, i.e., bids submitted by $p a_{i}$ in the RAGs should be rational due to its $F_{i}(x)$ according to theorem 1.

In $D M_{F}$, let $M$ represent the total number of resource allocation games $\left\{R A G_{1}, \ldots, R A G_{M}\right\}$ which $p a_{i}$ had participated in. Let $b_{i}^{m}$ represent the bid submitted by $p a_{i}$ in an auction $R A G_{m}(1 \leq m \leq M)$, which was also reported by the resource provider to $\mathrm{CA}$. Next, for each $b_{i}^{m}$, CA assumes that $p a_{i}{ }^{\prime} s$ bidding strategy in $R A G_{m}$ is optimal, and it derives $p a_{i}{ }^{\prime} s$ valuation $v_{i}^{m}=\beta^{-1}\left(b_{i}^{m}\right)$ according to theorem 1 . Then, each $v_{i}^{m}$ is viewed as a sample $Y_{m}$ in $D M_{F}$. Now CA has a group of such samples $\left\{Y_{1}, \ldots, Y_{M}\right\}$ with a size $M$. Finally, CA only needs to test whether the samples $\left\{Y_{1}, \ldots, Y_{M}\right\}$ follow $F_{i}$ on $[\underline{v}, \bar{v}]$.

To do this, CA starts by postulating two hypotheses:

- Null hypothesis $H_{0}:\left\{Y_{1}, \ldots, Y_{M}\right\}$ follows $F_{i}(x)$, which indicates that $p a_{i}$ honestly reports its $F_{i}(x)$;

- Alternative hypothesis $H_{1}:\left\{Y_{1}, \ldots, Y_{M}\right\}$ does not follow $F_{i}(x)$, which indicates that $p a_{i}$ misreports its $F_{i}(x)$.

CA then divides the interval $[\underline{v}, \bar{v}]$ on which the $\left\{Y_{1}, \ldots, Y_{M}\right\}$ distributes into $D$ disjoint intervals, $I_{1}, \ldots, I_{D}$. Let $O_{j}$ be the actual number of samples located in $I_{j}$, and $E N_{j}$ be the expected number of samples located in $I_{j}$, i.e., $E N_{j}=M \int_{I_{j}} f_{i}(x) d x$. (Here, $f_{i}(x)$ is the corresponding $p d f$ of $\left.F_{i}(x)\right)$. Then, CA obtains

$$
\chi^{2}=\sum_{j=1}^{D} \frac{\left(O_{j}-E N_{j}\right)^{2}}{E_{j}}
$$

If $M$ is large enough (i.e., $M \geq 50$ ), Eq.(4) will approximately follow $\chi^{2}(D-1)^{3}$. Let $\alpha$ be the significance level, that is,

$$
\operatorname{Pr}\left(H_{0} \text { is true but } H_{0} \text { is resused }\right)=\alpha \text {. }
$$

For $\chi^{2}$ in Eq.(4), if it has $\chi^{2} \geq \chi_{\alpha}^{2}(D-1)$, CA immediately refuses the hypothesis $H_{0}$, and $p a_{i}$ is identified to have misreported its $F_{i}(x)$.

In general, $D M_{F}$ can be used to detect whether a peer's bidding behavior is consistent with its $F_{i}(x)$.

\subsection{Peer excessively bids for resources}

In each stage, some malicious peers may submit bids for as many resources as possible, so that they can increase their probabilities of winning the resources, and acquire those resources at lower prices. This harmful behavior can greatly decrease the payoff gained by other peers.

Now, we propose another method $D M_{E}$ to prevent this behavior, which is also based on chi-square goodness-of-fit test. The idea of $D M_{E}$ is that, if $p a_{i}$ submits bids for more than one resource to finish its task 
$a_{i}$, then the valuations derived from these bids will not accurately follow its reported $F_{i}(x)$. As presented in Section 4.1, peers will have to honestly report their $F_{i}(x)$ and take the optimal bidding strategy. Then, CA only needs to check whether $p a_{i}{ }^{\prime} s$ bidding behavior is consistent with its reported $F_{i}(x)$.

Different from $D M_{F}$, in $D M_{E}$, we let $\left\{b_{i}^{m}\right\}$ be a set of bids submitted by $p a_{i}$, which are reported by resource providers to $\mathrm{CA}$, and $M$ be the size of $\left\{b_{i}^{m}\right\}$. Then, for each $b_{i}^{m}$, CA can immediately derive $p a_{i}{ }^{\prime} s$ valuation $v_{i}^{m}=\beta^{-1}\left(b_{i}^{m}\right)$ by theorem 1 . Next, each $v_{i}^{m}$ is viewed as a sample $Y_{m}$ in $D M_{E}$. Now, CA has a group of such samples $\left\{Y_{1}, \ldots, Y_{M}\right\}$, and it only needs to check whether the samples $\left\{Y_{1}, \ldots, Y_{M}\right\}$ is consistent with $p a_{i}{ }^{\prime} s$ $F_{i}(x)$ on $[\underline{v}, \bar{v}]$. To do this, CA postulates two hypotheses:

- Null hypothesis $H_{0}:\left\{Y_{1}, \ldots, Y_{M}\right\}$ are consistent with $F_{i}(x)$, which indicates that $p a_{i}$ does not excessively bid for resources ;

- Alternative hypothesis $H_{1}:\left\{Y_{1}, \ldots, Y_{M}\right\}$ are not consistent with $F_{i}(x)$, which indicates that $p a_{i}$ has the behavior of excessively bidding for resources.

If it finds $\chi^{2} \geq \chi_{\alpha}^{2}(D-1)$ (where $\alpha$ is the confidence interval), CA immediately refuses the hypothesis $H_{0}$, and $p a_{i}$ is detected for having behavior of excessively bidding for resources. We conduct experiments on the efficiency of $D M_{E}$, which are given in Section 6.2.

\section{Resource Allocation among Peers}

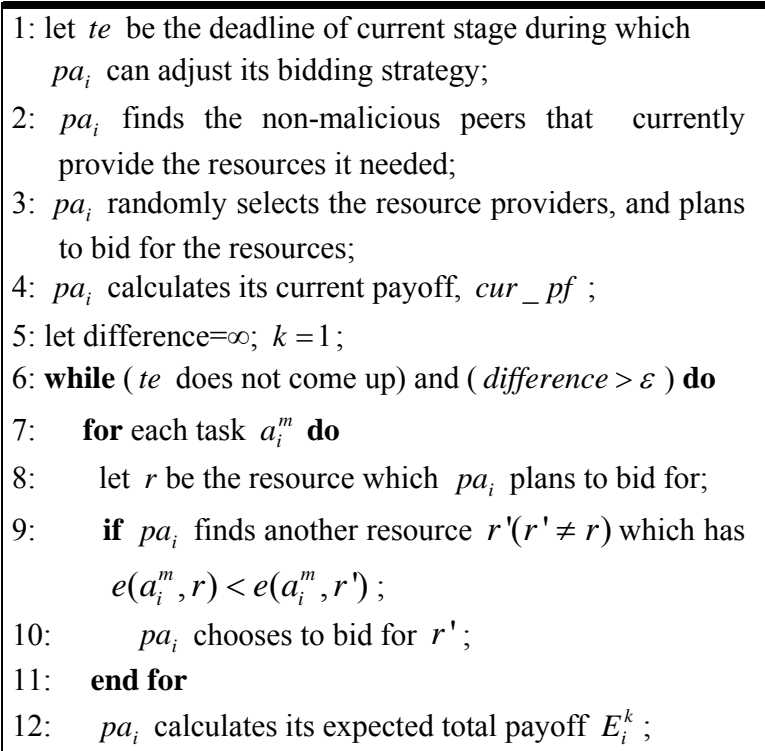

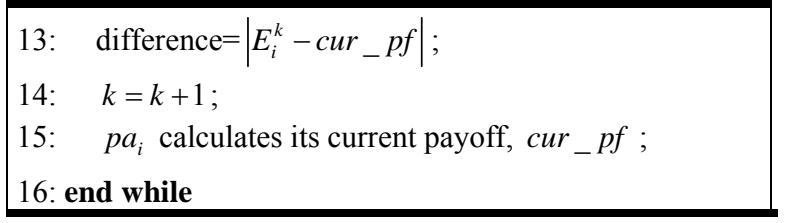

Fig. 1. Algorithm for helping $p a_{i}$ make its bidding strategy in stage $t$

In any stage, a peer may hold tasks and resources simultaneously. For each task, it bids for a corresponding resource to accomplish it. While for the resources, it sells them out by auction. In this section, we present an algorithm for helping each peer bid for the resources.

To analyze this process quantitatively, we use an indicator $x\left(a_{i}^{m}, r_{j}^{n}\right)$ to denote whether $p a_{i}$ can successfully obtain resource $r_{j}^{n}$ and finish its task $a_{i}^{m}$, that is, if $p a_{i}$ obtains $r_{j}^{n}$, then $x\left(a_{i}^{m}, r_{j}^{n}\right)=1$; otherwise, $x\left(a_{i}^{m}, r_{j}^{n}\right)=0$. Let $e\left(a_{i}^{m}, r_{j}^{n}\right)$ be the corresponding payoff gained by $p a_{i}$ in finishing $a_{i}^{m}$. Then, the total amount of payoff, $E_{i}(t)$, gained by $p a_{i}$ in $t$ is given by

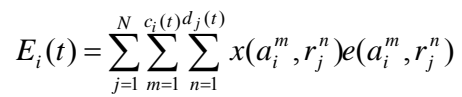

Let $E_{i}^{k}(t)$ denote the total payoff gained by $p a_{i}$ in the $k$-th adjustment on its bidding strategy. Then, for some $\varepsilon>0$ set by $p a_{i}$ itself, if it observes that

$$
\left|E_{i}^{k}(t)-E_{i}^{k+1}(t)\right|<\varepsilon
$$

$p a_{i}$ will not change its bidding strategy any more.

The algorithm for a peer to make its bidding strategies in time stage $t$ is given in Figure 1.

\section{Experiments}

In this section, we present experimental analysis on our resource allocation approaches. We test the effects of different parameters on the bidding results. The simulations are developed with $\mathrm{C}++$ programming language and Matlab 7.0. In the experiments, the number of peers is set to be $N=30$. Each peer's valuation, $F_{i}$, follows the uniform probability distribution on $[0,1]$, i.e., $v=0$ and $\bar{v}=1$. The number of tasks generated by one peer in each stage, $c_{i}(t)$, is randomly selected in $[1,20]$. In the experiments, we studied on the peers' payoff and their successfully bidding ratio $(S B R)$ (i.e., the ratio at which a peer $p a_{i}$ can successfully obtain the resources to finish its tasks). 
$S B R_{i}$ is defined by $S B R_{i}=\frac{\sum_{t=1}^{K} n r_{i}(t)}{\sum_{t=1}^{K} c_{i}(t)}$, where $K$ is the number of stages that the system has run, and $n r_{i}(t)$ is the number of resources obtained by $p a_{i}$ through bidding in stage $t$. Thus, if the value of $S B R_{i}$ is 1 , it means that $p a_{i}$ can always successfully obtain the resources in the auctions. If the value of $S B R_{i}$ is 0 , it means that $p a_{i}$ can obtain no resources in any RAG.

\subsection{Peer misreporting its $F_{i}(x)$}

In the experiments, we compared the average payoff and $S B R$ gained by each non-malicious peer under two different malicious scenarios, i.e., the scenario where the system uses $D M_{F}$ (proposed in Section 4.1) to detect the malicious behaviors (shown in figure 2(c) and (d)), and the scenario where the system does not use $D M_{F}$ (shown in figure 2(a) and (b)). The percent of malicious peers, $\omega$, is set to increase from $10 \%$ to $80 \%$. While using $D M_{F}$, the parameter $\alpha$ is increased from 0.02 to 0.08 , and the number of time stages is fixed to 1000. Each malicious $p a_{i}$ misreports its $F_{i}(x)$ as a uniform probability distribution on a different domain of $[0.8,1]$.

In the experiments, we record the ratio of nonmalicious peers' payoff and SBR with $\omega$ percent of malicious peers in the system, to their payoff and $S B R$ with no malicious peers in the system, respectively. As shown in Eq.(7) and (8), the ratio of non-malicious participants' payoff and $S B R$ are denoted by $\eta_{\omega}($ payoff $)$ and $\eta_{\omega}(S B R)$, respectively.

$$
\eta_{\omega}(\text { payoff })=\frac{\sum_{t=1}^{K} \sum_{i \in \Theta_{\omega}} E_{i}^{n}(t)}{\sum_{t=1}^{K} \sum_{i=1}^{n} E_{i}(t)}
$$

We let $S B R_{i}^{n}(t)$ be the average amount of $S B R$ gained by a non-malicious $p a_{i}$ in stage $t$ when the environment contains some malicious peers.

$$
\eta_{\omega}(S B R)=\frac{\sum_{t=1}^{K} \sum_{i \in \Theta_{\omega}} S B R_{i}^{n}(t)}{\sum_{t=1}^{K} \sum_{i=1}^{n} S B R_{i}(t)}
$$

In the equations, we use $\Theta_{\omega}$ to denote the set of nonmalicious peers in the environment which contains $\omega$ percent of malicious peers, $E_{i}^{n}(t)$ to denote the amount of payoff gained by a non-malicious $p a_{i}$ under the malicious scenario in time stage $t$, and $E_{i}(t)$ to represent its payoff in stage $t$ under the scenario with no malicious behaviors.

As shown in figure 2 (a) and (b), when $\omega$ increases, the values of $\eta_{\omega}(S B R)$ and $\eta_{\omega}$ (payoff) are reduced greatly. This is because the non-malicious peers bidding at rational prices have little chance to obtain the resources in the auctions with their valuations distributed on $[0,1]$. However, when the system uses $D M_{F}$ detect the cheating behaviors, the malicious peers will be very careful to submit their bids so as to avoid being detected out. In this way, the non-malicious peers can gain more payoff and $S B R$. For example, even if the $\omega$ increases up to 0.8 , the non-malicious peers can obtain more than 96 percent of the payoff that they may obtain in a non-malicious scenario.

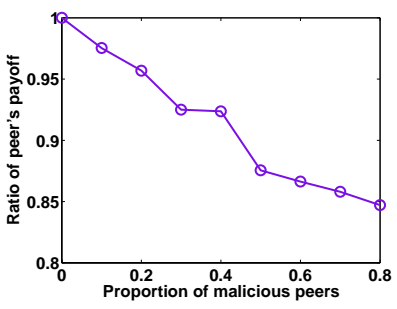

(a) Peer's payoff with no $D M_{F}$

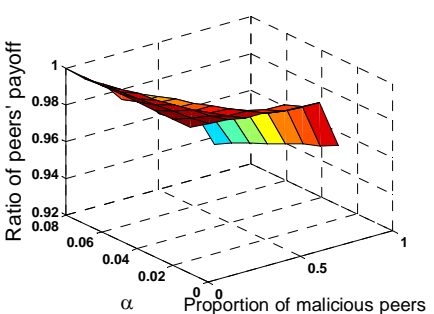

(c)Peer's payoff by using $D M_{F}$

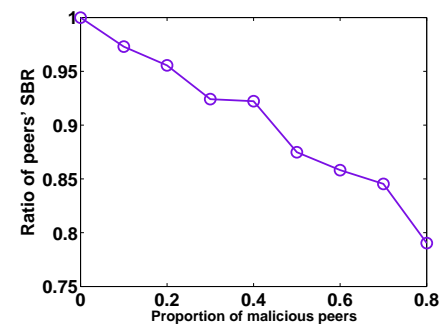

(b) Peer's $S B R$ with no $D M_{F}$

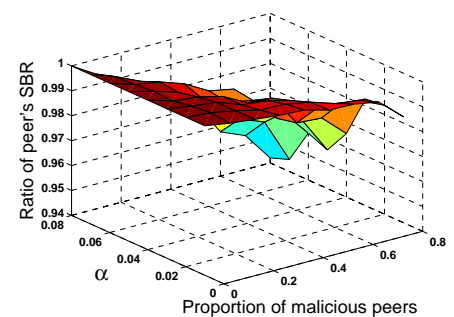

(d) Peer's $S B R$ by using $D M_{F}$
Fig. 2. Detecting peers' designed malicious behavior

\subsection{Peers excessively bidding for resources}

In this section, we use $D M_{E}$ to identify the malicious behavior of excessive bidding. We set $\omega$ (the percent of malicious peers) to increase from $10 \%$ to $80 \%$. In every time stage $t$, a malicious $p a_{i}$ generates $c_{i}(t)$ tasks, and it will bids for $\pi c_{i}(t)(\pi>1)$ resources. The value of $\pi$ increases from 2 to 4 . To identify this malicious behavior, we use the equation $\chi^{2}=\sum_{j=1}^{D} \frac{\left(O_{j}-E N_{j}\right)^{2}}{E_{j}}$ to check each $p a_{i}{ }^{\prime}$ s action. The parameter $\alpha$ is set as 0.05 , and the value of $D$ varies in the set of 
$\{10,20,30,40,50,60,70,80,90,100\}$. The value of $M$ is set to 2000 .

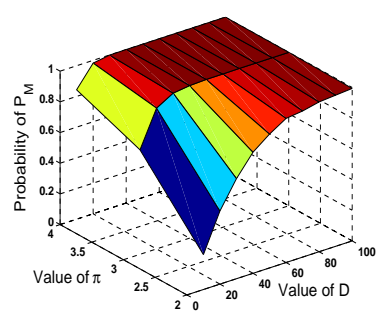

(a) Probability of detecting malicious peers

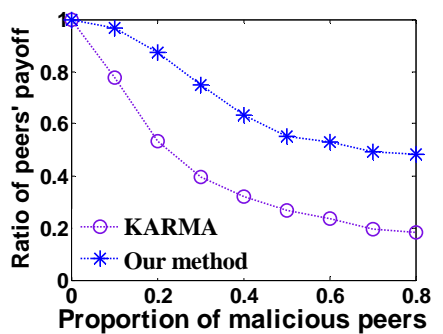

(c) Peer's payoff under two methods

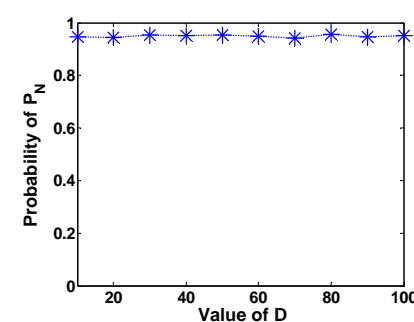

(b) Probability of confirming non-malicious peers

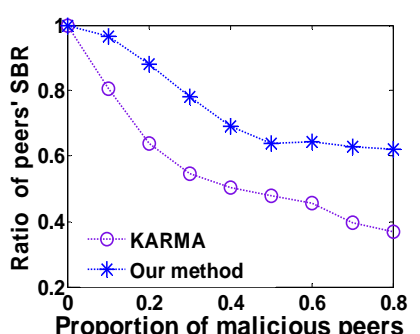

(d) Peer's SBR under two methods
Fig. 3. Peers excessively bidding for resources

To demonstrate the effectiveness of $D M_{E}$, we focus on two aspects, i.e., whether $D M_{E}$ can effectively identify the malicious peers that excessively bid for resources, and whether $D M_{E}$ is too sensitive to mistreat the non-malicious peers as malicious ones. Thus, two probabilities $P_{M}$ and $P_{N}$ are analyzed. $P_{M}$ represents the probability that a malicious peer can be detected out by $D M_{E}$, while $P_{N}$ denotes the probability that a nonmalicious peer will not be detected out as a malicious one in $D M_{E}$. As shown in figure 3 (a) and (b), when $\pi$ increases, the value of $P_{M}$ is incremented significantly, which means that $D M_{E}$ becomes more accurate in detecting the malicious peers. The reason is that when a peer submits bids for more resources than it actually needs, its bidding behavior will deviate significantly from its $F_{i}(x)$. We can also observe from figure 3(b) that the probability $P_{N}$ is approximately 0.945 , and the value of $D$ has little impact on it. This means that $D M_{E}$ seldom regards a non-malicious peer as a malicious one in performing excessively bidding for resources. In this way, we show the effectiveness of $D M_{E}$.

To show the performance of our method, we also compare it with another method named KARMA ${ }^{16}$, which also implemented banks to facilitate secure auctions so as to resist the malicious behavior. In the experiments, the number of resources that each malicious peer bids for is four times as many as the number of tasks it generates. The results are shown in figure 3 (c) and (d). We can find that the payoff and SBR of non-malicious peers suffer greatly from this type of malicious behavior. We can also see that our method performs much better than KARMA. The reason is that if each malicious peer will pay for every resource that it has won, KARMA can not find the corresponding malicious behavior of excessively bidding for resources. While in our method, if a malicious peer submits bids for more resources than it actually needs, the valuations derived from these bids will not accurately follow its $F_{i}(x)$. Thus, our method can detect this malicious behavior.

\section{Conclusion}

In a peer-to-peer system, peers may tend to be selfish for saving their limited resources and act as the freeriders. Some peers may even be malicious with the goal to damage the system. To address these issues, we present a market-based approach for allocating resources to autonomous peers holding prioritized tasks, and detecting malicious peers in non-cooperative peerto-peer environments. Several statistical mechanisms are presented for detecting out the malicious peers from the non-cooperative environments. Finally, we conduct some experiments to show the effectiveness of the proposed mechanisms.

\section{Acknowledgements}

This work is supported by the National Natural Science Foundation of China under Grant No.60903053, the Natural Science Foundation of Zhejiang Province(Z1100822, LY12F02005), the Open Foundation of State Key Laboratory for Novel Software Technology of Nanjing University (KFKT2011B07).

\section{References}

1. M. Karakaya, I. Korpeoglu and O. Ulusoy, Free Riding in Peer-to-Peer networks, IEEE Internet Comput., 13(2) (2009), pp.92-98.

2. M. J. Osborne, Introduction to Game Theory (Oxford University Press, Oxford, 2004).

3. R. B. Ash, Basic Probability Theory (Dover publication, New York, 2008).

4. J. B. Rosen, Existence and uniqueness of equilibrium points for concave n-person games, Econometrica 33(3)(1965), pp.520-534.

5. Y. Qiu and P. Marbach, Bandwidth allocation in Ad Hoc 
networks: a price-based approach, in Proc. 22nd Int. Conf. IEEE Computer and Communications Societies, eds. F. Bauer and J. Roberts (San Franciso, USA, 2003), pp. 797-807.

6. C. Curescu and S. N. Tehrani, A bidding algorithm for optimized utility-based resource allocation in Ad Hoc networks, IEEE Trans. Mob. Comput., 7(12) (2008), pp.1397-1414.

7. T. Guang and S. S. Jarvis, A payment-based incentive and service differentiation scheme for Peer-to-Peer streaming broadcast, IEEE Trans. Parallel Distrib. Syst., 19(7) (2008), pp.940-953.

8. Y. Yonghe, A. E. Atawy and E. A. Shaer, Ranking-based optimal resource allocation in Peer-to-Peer networks, in Proc. 26th Int. Conf. IEEE Computer and Communications Societies, eds. R. L. Baldwin and G. Kesidis (Anchorage, Alaska, USA, 2007), pp. 1100-1108.

9. J. Sonnek, A. Chandra and J. B. Weissman, Adaptive reputation-based scheduling on unreliable distributed infrastructures, IEEE Trans. Parallel Distrib. Syst., 18(11) (2007), pp.1551-1564.

10. A. Satsious and L. Trssiulas, Reputation-based resource allocation in P2P system of rational users, IEEE Trans. Parallel Distrib. Syst., 21(4) (2010), pp.466-479.

11. M. Feldman, K. Lai and L. Zhang, The proportionalshared allocation market for computational resources, IEEE Trans. Parallel Distrib. Syst., 20(8) (2009), pp.1075-1088.

12. M. Felegyhazi, J. P. Hubaux and L. Buttyan, Nash equilibria of packet forwarding strategies in wireless $\mathrm{Ad}$ Hoc networks, IEEE Trans. Mob. Comp., 5(5) (2006), pp.463-476.

13. W. Yu and H. J. R. Liu, Game theoretic analysis of cooperation stimulation and security in autonomous mobile Ad Hoc networks, IEEE Trans. Mob. Comput., 6(5) (2007), pp.507-521.

14. H. Nishida and T. Nguyen, A global contribution approach to maintain fairness in $\mathrm{P} 2 \mathrm{P}$ networks, IEEE Trans. Parallel Distrib. Syst., 21(6) (2009), pp.812-826.

15. M. Z. Jin, X. Lei, and J. Du, Evolutionary game theory in multi-objective optimization problem, Int. J. Comput. Intell. Syst., 3(s1)(2010), pp.74 - 87.

16. S. C. V. Vishnumurthy and E. G. Sirer, KARMA: A Secure Economic Framework for P2P Resource Sharing, in Proc. 2nd Int. Conf. Economics of Peer-To-Peer Systems, eds. M. F. Kaashoek and I. Stoica (Berkeley, CA, USA, 2003), pp.1-6.

17. D. Banerjee, S. Saha, S. Sen, and P. Dasgupta, Reciprocal Resource Sharing in P2P Environments, in Proc. 4th Int. Conf. Autonomous Agents and Multi-Agent Systems, eds. F. Dignum and S. Koenig (Utrecht, The Netherlands, 2005), pp. 853-859.

18. Y. Chu, J. Chuang and H. Zhang, A Case for Taxation in Peer-to-Peer Streaming Broadcast, in Proc. 2nd Int. Conf. Practice and Theory of Incentives and Game Theory in Networked Systems, eds. R. Yavatkar, E. W. Zegura and
J. Rexford (Portland Oregon, USA, 2004), pp. 205-212. 